\title{
Conciliación familia-trabajo y sintomatología externalizante de los hijos e hijas: papel mediador del clima familiar
}

\author{
Ana Martínez-Pampliega*, Itxaso Ugarte, Laura Merino y David Herrero-Fernández \\ Universidad de Deusto
}

- Recibido: 24 - 10 - 2017 - Aceptado: 08 - 10 - 2018 . Avance online: 21 - $11-2018$

RESUMEN: El objetivo de este estudio es analizar la relación existente entre las dificultades de conciliación familia-trabajo y la sintomatología externalizante de los hijos e hijas, analizando el papel mediador de las variables familiares. Estos ámbitos de estudio rara vez han sido abordados de forma conjunta. En el estudio han participado 74 adolescentes entre 14 y 16 años, quienes proporcionaron respuestas sobre las siguientes variables: conciliación laboral-familiar, funcionamiento familiar, estilos parentales, conductas agresivas y conductas de ruptura de normas. Las dificultades de conciliación se asociaron de forma significativa con la presencia de conductas de ruptura de normas en los hijos/as, siendo esta relación mediada totalmente por las variables comunicación y supervisión familiar. Aunque los resultados deben tomarse con cautela, apuntan hacia la importancia de los mecanismos familiares como vía de profundización en la comprensión del impacto de las dificultades de conciliación en la sintomatología de los hijos e hijas, concretamente sintomatología externalizante.

PALABRAS CLAVE: Conciliación Familia-Trabajo, Estilos Parentales, Clima Familiar, Sintomatología Externalizante.

Work-family balance and children's external simptomatology: the mediating role of family climate ABSTRACT: The aim of this study is to analyze the relationship between the difficulties of work-family balance and children's externalizing symptomatology by studying the mediating role of family variables. These areas of study have rarely been addressed together. The study involved 74 adolescents between 14 and 16 years old who provided answers about the following variables: work-family balance, family functioning (cohesion, adaptability, family satisfaction, family communication), parenting styles, aggressive and rupture rule behavior. The difficulties of balance were significantly associated with the presence of rupture rules behaviors in children, and this relation fully mediated by family communication and monitoring variables. The mechanisms seem to provide a potential family-depthway to understand the impact of the difficulties of work-family balance on the symptomatology of children, especially externalizing. While these results should be approached with caution, they illustrate the need to continue to deepen the understanding of this research line. KEYWORDS: Work-Family Balance, Parenting Styles, Family comunication, Family Climate, Externalizing Symptomatology.

Tradicionalmente, madres y padres han jugado diferentes roles en el sistema familiar (Finley, Mira y Schwartz, 2008). Sin embargo, en las últimas décadas esta división tradicional, en la cual el marido es el soporte económico de la familia y la mujer es el soporte

*Correspondencia: Ana Martínez-Pampliega.

Universidad de Deusto.

Código Postal: 48007, Bilbao, España.

E-mail: martinez.pampliega@deusto.es doméstico, está cambiando de diferentes formas dependiendo del país y la cultura (Ababkov, Perrez, Kaindanovskaia y Shiobi, 2005). Hay una tendencia global hacia una mayor participación de la mujer en el mundo laboral (Aryee, Srinivas y Tan, 2005) y un incremento del número de parejas en las que ambos miembros trabajan. Al mismo tiempo, se observa un mayor incremento de familias con un solo progenitor.

Uno de los ámbitos en los cuales se observa el impacto de la incorporación laboral de la mujer es en el clima familiar, es decir, en 
la crianza y en el funcionamiento familiar, lo que ha desembocado en un interés mayor por investigar cómo los miembros de estas nuevas familias organizan sus responsabilidades familiares y laborales, o lo que es lo mismo, cómo concilian su vida familiar y laboral. Se ha puesto de manifiesto la visible tensión entre ambos mundos (Carrasco, 2001): las exigencias de mayor flexibilización, de mayor disponibilidad y movilidad exigidos por las empresas a sus trabajadores, vs. el derecho de los trabajadores a su vida familiar, a poder cuidar de sus hijos y disfrutar de la vida familiar junto a ellos (López, Utrilla y Valiño, 2006).

Las investigaciones recientes tratan de analizar el impacto que esta tensión puede tener en la vida familiar y en el clima emocional (Ford, Heinen y Langkamer, 2007; Gonzalo, 2016; Honeycutt, Sheldon, Pence, y Hatcher, 2016). Esta relación entre trabajo y familia es bidireccional, es decir, el trabajo puede influir en la vida familiar o, al revés, la familia puede interferir en la vida laboral. Spector et al. (2007) exponen que los individuos suelen acusar mayor interferencia del trabajo en la familia que al revés. Por ello, y dada su importante vinculación con las pautas de crianza nos centraremos en el conflicto trabajo-familia.

Estos conflictos experimentados pueden tomar diferentes formas dependiendo de si están basadas en el tiempo o en la tensión. En función del tiempo, una larga jornada de trabajo, la existencia de turnos, las horas extras, pueden generar presión en la vida familiar (Ford et al. 2007). Algunos autores se refieren a esto como sobrecarga del rol y la describen como la percepción de tener muchas cosas que hacer y no tener tiempo suficiente para hacerlas (Ayree et al. 2005; Alpert, 2010).

Esta sobrecarga afecta al nivel de implicación de los progenitores en el cuidado de los hijos e hijas, influyendo directamente en las responsabilidades que asume el otro progenitor en su cuidado y en el porcentaje de tiempo en el que éste actúa como cuidador principal o supervisor (Jacobs y Kelley, 2006; Matias, y Fontaine, 2014). En este sentido, en un estudio realizado en Singapur, Skitmore y Ahmad (2003) encontraron que cerca de un $40 \%$ de los participantes con hijos/as en edad preescolar o escolar raramente o nunca atienden a las actividades de sus hijos/as y muchos responsabilizaron a la falta de tiempo su incapacidad para atenderlos.

También esta sobrecarga conlleva fatiga y agotamiento afectando negativamente a las demandas familiares, al estado anímico y a las reacciones emocionales $y$, por tanto, contribuye indirectamente a desarrollar valoraciones más negativas sobre la familia (Ilies et al., 2007) y a generar mayor insatisfacción familiar. Esto tiene lugar, de acuerdo con los autores citados, incluso aunque la sobrecarga no sea crónica ni objetiva. Lo relevante es la percepción subjetiva experimentada. A su vez, en dicho estudio se observó que los estados afectivos que los participantes experimentaron en su trabajo tendían a persistir después de ir a casa, tras acabar la jornada de trabajo. Estos sentimientos negativos, parecen mediar el impacto que la sobrecarga tenga en casa. Además, es importante considerar que este conflicto es aún mayor en aquellas parejas en las que ambos miembros trabajan fuera de casa (Livingston y Judge, 2008).

La otra forma que puede tomar el conflicto familia-trabajo y que recalcan Golden, Veiga, y Simsek (2006), ha sido la basada en la tensión, la cual puede derivarse de tres fuentes principales (Scharlach, 2001): (1) una gran carga de demandas familiares, (2) grandes demandas del trabajo, (3) pobre apoyo familiar (acuerdos inadecuados en relación al cuidado de los hijos/ as o ausencia del otro progenitor) o laboral.

Esta tensión genera baja satisfacción familiar, gran hostilidad en casa y una variedad de consecuencias psicológicas cuando el grado de conflicto es alto (llies et al., 2007), lo cual a su vez provoca una disminución del número de actividades sociales que el/la trabajador/a realiza con su cónyuge e hijos/as. Ilies et al. (2007) encontraron que los y las empleados/as que describían un alto grado de conflicto trabajofamilia eran menos propensos a interaccionar socialmente con sus familias, incluso cuando se había controlado la cantidad de tiempo que el empleado pasaba en casa.

En conclusión, ya sea por falta de tiempo o por tensión, se observa sobrecarga emocional y disminución de la implicación, lo cual afecta a la vida familiar. No obstante, la vinculación entre las tres variables, dificultades en la conciliación familiar-laboral, clima familiar, y sintomatología externalizante de los hijos e hijas 
no ha sido muy estudiada. Con todo ello, en este estudio se hipotetiza que el conflicto familiatrabajo podría afectar a la sintomatología de los y las adolescentes a través del impacto que el conflicto trabajo-familia tiene en el clima familiar. Existen diferentes planteamientos sistémicos, que podrían contribuir a explicarlo: (1) La hipótesis de la transferencia: la hostilidad manifestada por los adultos en torno a su trabajo podría afectar negativamente a las relaciones familiares. Tendríamos que presuponer que a mayor hostilidad manifestada hacia el trabajo mayor hostilidad manifestada en las relaciones familiares, haciendo una extrapolación de los resultados sobre el papel de la hostilidad encontrado en los estudios de Low y Stocker (2005). (2) Las prácticas de crianza. De acuerdo con esta hipótesis, los conflictos afectan de forma negativa en las prácticas de crianza utilizadas por los progenitores y en la consistencia de su aplicación. Esto podría producirse por: (a) el incremento del estrés de los padres y las madres; Serrano-Manriquez (2007) señala en su estudio que aquellos que presentaban una mayor vulnerabilidad al estrés laboral, ejercían unas prácticas de crianza muy autoritarias, llegando incluso a acciones como humillación, críticas, descalificaciones,...; (b) las mayores discusiones interparentales y su repercusión en los hijos e hijas, a través de un estilo de disciplina más negativo y una mayor inconsistencia en la aplicación de la disciplina. En estos casos, las discusiones se generan en torno a temas vinculados con los hijos/as, por lo que pueden provocar la sensación en éstos/as de que son ellos los responsables de la discusión. (3) Las relaciones afectivas entre padres, madres e hijos/as: El agotamiento emocional que genera la tensión del trabajo agota los recursos de los progenitores para responder ante las necesidades de los hijos e hijas y, a su vez, se muestran menos afectuosos/as con ellos/as. Esto ha sido relacionado con conductas, fundamentalmente, de carácter antisocial (Crum y Moreland, 2017; Davies y Cumming, 1994; Unger, Brown, Tressell y Ellis, 2000). Las experiencias acumuladas de hostilidad pueden hacer que primen las evaluaciones y atribuciones negativas sobre la conducta del hijo o hija o que se trastoque la habilidad de percibir sus necesidades.

El objetivo de este artículo es profundizar en la relación entre la conciliación entre la vida familiar y laboral, los estilos educativos y la sintomatología externalizante de los hijos/ as, centrándonos fundamentalmente en los comportamientos agresivos y las conductas de ruptura de normas de los y las adolescentes. En esta relación, se tendrá en cuenta el papel mediador del clima familiar (funcionamiento familiar y estilos parentales).

\section{MÉTODO}

\section{-PARTICIPANTES}

Este estudio se desarrolló con la participación de 74 adolescentes $(39.2 \%$ chicos y $60.8 \%$ chicas) con edades comprendidas entre 14 y 16 años $(M=15.01$; D.T. $=.69)$ pertenecientes a diversos cursos de Educación Secundaria Obligatoria de un centro educativo de Bilbao.

Las familias de los y las adolescentes que participaron en este estudio estaban compuestas en un $83.8 \%$ por ambos progenitores, solamente un $16.2 \%$ refirió vivir sólo con la madre y hermanos/as. Un 64.9\% del total indicó tener al menos un hermano/a. En la mayoría de estas familias ambos progenitores son trabajadores (73\%). Los participantes procedían de 6 de los 8 distritos de Bilbao.

\section{•INSTRUMENTOS}

- Conciliación: Construido ad-hoc para el estudio. Se trata de un cuestionario que recoge a través de 7 preguntas $(\alpha=.84)$, la sobrecarga o tensión experimentada por los padres y madres en el trabajo (llegar a casa tarde y sin terminar, trabajar en casa, volver nerviosos/as, enfadados/as o cansados/as, quejarse del trabajo). Se trata de un instrumento unidimensional al cual se responde a través de una escala likert de 5 puntos, desde 1 (nunca) a 5 (muy frecuentemente). Ver tabla 1.

- FACES 20-Esp (Escala de Evaluación de la Adaptabilidad y de la cohesión familiar) desarrollado por Olson, Portner y Bell (1982). EI FACES 20-Esp constituye la versión breve y adaptada culturalmente en España por Martínez-Pampliega, Iraurgi, Galíndez y Sanz, (2006), de la escala americana "Family adaptability and cohesión evaluation scale. 
Tabla 1

Estadísticos descriptivos, análisis de fiabilidad y análisis factorial del cuestionario de Conciliación

\begin{tabular}{|c|c|c|c|c|c|}
\hline ¿Con qué frecuencia & M & $D T$ & $r$ & $\alpha$ & $\begin{array}{r}\text { Análisis } \\
\text { factoria }\end{array}$ \\
\hline 1- Has tenido que cambiar de planes por el trabajo de tus padres? & 1.99 & .96 & .45 & .84 &, 594 \\
\hline 2 - Llegan tarde a casa y no les da tiempo a hacer todo lo que quisieran? & 2.44 & 1.01 & .60 & .82 & ,701 \\
\hline 3 - Llegan nerviosos del trabajo? & 2.58 & 1.08 & .64 & .82 & ,738 \\
\hline 4 - Siguen trabajando una vez en casa? & 2.60 & 1.36 & .51 & .84 &, 579 \\
\hline 5 - Llegan tus padres enfadados del trabajo? & 2.15 & .90 & .70 & .81 & ,796 \\
\hline 6 - Se quejan tus padres de su trabajo? & 2.36 & 1.06 & .64 & .82 & ,688 \\
\hline 7 - Llegan tus padres tarde y cansados a casa? & 3.05 & 1.16 & .73 & .80 & ,791 \\
\hline $\begin{array}{l}\mathrm{KMO}=.853 ; \chi^{2}=340.147 ; p<.001 \\
\text { Varianza explicada }=51 \%\end{array}$ & 2.45 & 1,07 & -- & .84 & \\
\hline
\end{tabular}

FACES II" (Olson et al. 1982). Este instrumento está basado en uno de los modelos con mayor relevancia actualmente en la comprensión de los sistemas familiares, el modelo Circumplejo. Estudia las dos dimensiones fundamentales del funcionamiento familiar: la cohesión y la adaptabilidad, teniendo en cuenta múltiples parámetros en cada una de estas variables. La escala consta de 20 ítems y cuenta con índices elevados de validez y fiabilidad. La versión española presenta unos índices alpha de Cronbach de .89 y .87 para cohesión y adaptabilidad respectivamente. A su vez, presentó una buena validez convergente con constructos afines.

- Escala de Satisfacción familiar. Esta escala fue elaborada por Olson en 1989 (Olson, Stewart y Wilson, 1990), basándose en la escala del mismo nombre, desarrollada por Olson y Wilson en 1982. Este instrumento intenta cubrir una de las hipótesis del modelo Circumplejo la cual señala que es más importante la satisfacción que experimenta una familia sobre su nivel de cohesión y adaptabilidad, que dónde está situada en el modelo. Originalmente esta escala constaba de 14 ítems, pero posteriormente en 1989, Olson la redujo a únicamente 10 (Olson et al., 1990), con los cuales se evalúa el grado de satisfacción experimentado con aspectos relacionados con la cohesión y adaptabilidad familiar. Esta escala obtuvo altos índices de fiabilidad tanto en la versión original (Olson y Wilson, 1982), con un alpha de Cronbach de .91 y un coeficiente de test- retest de .75, como en la adaptación española (Sanz, 2003; Sanz, Iraurgi y Martínez-Pampliega, 2002), presentando un alpha de Cronbach de .92 y un coeficiente de test- retest de .95.

- Comunicación familiar. Esta escala fue creada por Barnes y Olson (1982) con el fin de cubrir la tercera dimensión del modelo Circumplejo y evaluar la comunicación que se produce entre progenitores y sus hijos/ as, teniendo en cuenta la apertura o libertad para intercambiar ideas, la información y las preocupaciones entre generaciones, la confianza y honestidad experimentada, y el tono emocional de las interacciones. El test original consta de 20 ítems y ha mostrado buenos índices de fiabilidad (alpha de Cronbach de .88 y coeficiente test-retest de.88), posteriormente se desarrolló una versión breve de tan sólo 10 ítems que estudian la comunicación global familiar basándose en las dimensiones de la escala original (Barnes y Olson, 1985). En este estudio se emplea la versión adaptada de la escala breve (Sanz et al., 2002), la cual ha demostrado una buena fiabilidad $(\alpha=.88)$.

- Alabama Parenting Questionnaire (Frick, 1991). El APQ mide las prácticas parentales 
a través de cinco subescalas: monitorización parental y supervisión, castigo inconsistente, castigo corporal, crianza positiva, desarrollo parental y otras prácticas disciplinarias (Dadds, Fraser y Maujean, 2003). Consta de 42 ítems presentados con una escala de frecuencia de cinco puntos: nunca, casi nunca, a veces, casi siempre y siempre. Estos autores en su estudio informan de una alta consistencia interna y un buen coeficiente test-retest para las subescalas. Es este estudio se utilizará la versión en español desarrollada por Servera (2007), centrándonos en las siguientes dimensiones: implicación parental, crianza positiva, disciplina inconsistente, pobre supervisión y disciplina severa. Las subescalas empleadas demostraron tener una fiabilidad entre moderada y alta en este estudio ( $\alpha=$ entre .70 y .85).

- Youth Self-Report (YSR). Se trata de un instrumento de autoinforme desarrollado por Achenbach (1991) para obtener información sistematizada directamente de niños y adolescentes (entre 11 y 18 años) sobre diversas competencias y problemas de conducta, a través de 112 ítems. Se ha propuesto una estructura factorial de primer y segundo orden para los cuadros psicopatológicos infanto-juveniles. Los dos factores de segundo orden son Síndrome Internalizado y Síndrome Externalizado. El Síndrome Internalizado incluye la Depresión/ Ansiedad, Quejas Somáticas y Problemas de Relación. El Síndrome Externalizado agrupa cuadros como la Búsqueda de Atención, las Conductas Delictivas y las Conductas Agresivas. El YSR posee una alta consistencia interna tanto para chicas como para chicos adolescentes (en escalas de externalización, alpha de cronbach de .90 y .88 para chicos y chicas respectivamente, Lohman, Newman y Newman, 2007).

\section{-PROCEDIMIENTO}

Tras el visto bueno del equipo directivo, se informó a los progenitores sobre la investigación, garantizando la confidencialidad y el anonimato. Los padres firmaron el consentimiento informado. Se llevó a cabo mediante una aplicación colectiva en la propia aula y bajo la supervisión de personal docente e investigador.

\section{• DISEÑO Y ANÁLISIS DE DATOS}

Por parte del diseño, se trata de un estudio transversal correlacional. Los análisis estadísticos se llevaron a cabo en dos partes. En una primera parte se analizaron las relaciones bivariadas entre todas las variables por medio del coeficiente de correlación de Pearson. En base a los resultados obtenidos se llevó a cabo el análisis de mediación. Este modelo se valoró mediante la significación del efecto total (relación entre variable independiente y variable dependiente, sin considerar el efecto de los mediadores), el efecto directo (relación entre la variable independiente y la variable dependiente, controlando el efecto de las variables mediadoras) y el efecto indirecto (diferencia entre efecto total y efecto directo controlando cada una de las variables mediadoras por separado y en conjunto). En el caso de efecto indirecto, se calculó el intervalo de confianza al $95 \%$ mediante la técnica de bottstrapping realizada sobre el re-muestreo con 5000 sub-muestras para determinar la existencia de mediación significativa o no. En el caso de que dicho intervalo de confianza no incluya el valor 0 , se concluye la existencia de mediación. Igualmente se calculó el total de varianza explicada de la variable independiente por las variables independiente y mediadoras, así como el tamaño de efecto de la mediación. Esto último se valoró a través del estadístico f2, que fue interpretado según el criterio de Cohen: valores de en torno a .02 son considerados pequeños; en torno a .15 medianos, y en torno a .35 o superior, grandes (Cohen, 1988). Para este análisis se empleó la macro Process 2.16 para SPSS (Hayes, 2013).

\section{RESULTADOS}

Primeramente, se analizó la asociación entre las variables relativas a conciliación familia - trabajo y el clima familiar y la sintomatología externalizante. Los resultados se detallan en la Tabla 2.

Tal como se puede observar, al estudiar la conciliación en relación al funcionamiento familiar se encontraron correlaciones moderadas y negativas entre falta de conciliación y, sobre todo, la variable comunicación. El continuar trabajando en casa, la insatisfacción en el trabajo, el cansancio, el nerviosismo se relaciona 
Tabla 2

Descriptivos, fiabilidad y correlaciones entre las variables del estudio

\begin{tabular}{|c|c|c|c|c|c|c|c|c|c|c|c|c|c|c|c|c|c|}
\hline & $M$. & D.T. & Intervalo & $\alpha$ & 1 & 2 & 3 & 4 & 5 & 6 & 7 & 8 & 9 & 10 & 11 & 12 & 13 \\
\hline 1. Conciliación & 17,18 & 5,42 & $5-35$ & .84 & 1 & & & & & & & & & & & & \\
\hline 2. Cohesión & 34,79 & 7,03 & $10-50$ & .86 & 要, 189 & 1 & & & & & & & & & & & \\
\hline 3. Adaptabilidad & 32,21 & 5,82 & $10-50$ & .76 & - 200 & ,787** & 1 & & & & & & & & & & \\
\hline 4. Com. Fam & 34,03 & 7,18 & $10-50$ & .88 &,$- 367^{* *}$ & ,719** & ,648" & 1 & & & & & & & & & \\
\hline 5. Satisf & 35,87 & 6,74 & $10-50$ & .89 &,$- 276^{*}$ & ,755* & ,618** & ,748** & 1 & & & & & & & & \\
\hline $\begin{array}{l}\text { 6. Implicación } \\
\text { parental }\end{array}$ & 53,93 & 11,72 & $19-95$ & .84 & ,006 & .664" &, $569^{* *}$ & , 358"* & , 485" & 1 & & & & & & & \\
\hline $\begin{array}{l}\text { 7. Crianza } \\
\text { positiva }\end{array}$ & 18,90 & 5,48 & $6-30$ & .85 &,- 224 & ,680** & ,614" &, $522^{* *}$ &, $567^{7 *}$ & .690** & 1 & & & & & & \\
\hline $\begin{array}{l}\text { 8. Disc. } \\
\text { Apropiada }\end{array}$ & 12,42 & 3,60 & $6-30$ & .56 &,$- 410^{* *}$ & $.315^{* *}$ &, $268^{*}$ & ,239* &, $255^{\star}$ & 138 &, $236^{*}$ & 1 & & & & & \\
\hline $\begin{array}{l}\text { 9. Disc. } \\
\text { Inconsistente }\end{array}$ & 14,09 & 4,28 & $6-30$ & .70 & ,279 & ,045 & ,021 & ,015 & ,040 &,- 028 & ,063 & ,060 & 1 & & & & \\
\hline $\begin{array}{l}\text { 10. Pobre } \\
\text { supervisión }\end{array}$ & 22,86 & 6,92 & $10-50$ & .79 &, $511^{* *}$ &,- 212 &,$- 246^{*}$ &,$- 343^{* *}$ &,$- 263^{*}$ &,- 113 &,- 230 &, $284^{*}$ &, $451^{* *}$ & 1 & & & \\
\hline 11. Disc. severa & 7,64 & 3,30 & $4-20$ & .82 & ,299*** &,$- 264^{*}$ &,$- 257^{*}$ & - 156 &,$- 265^{*}$ & 年7 & - 207 &, $540^{* * *}$ & 192 & 196 & 1 & & \\
\hline 12. Agresividad & 27,39 & 4,44 & $18-54$ & .76 & ,212 &,$- 294^{*}$ &,$- 315^{* *}$ & $-360^{* *}$ &,$- 348^{* *}$ &,- 127 & 年 &, $262^{*}$ & 107 & , 232* & , $440^{* *}$ & 1 & \\
\hline $\begin{array}{l}\text { 13. Ruptura de } \\
\text { normas }\end{array}$ & 21,19 & 3,91 & $14-42$ & .75 &, $426^{* *}$ &,$- 456^{* *}$ & - $439^{* *}$ & $-541^{* *}$ &,$- 563^{* *}$ &,- 123 &,- 207 & ,379** & , 139 & ,515** & , $382^{* *}$ &, $534^{* *}$ & 1 \\
\hline
\end{tabular}

Nota. ${ }^{*} p<.01{ }^{* *} p<.001$

con una peor comunicación entre los miembros de la familia.

Correlaciones igualmente significativas e incluso de mayor tamaño fueron encontradas con respecto a los estilos parentales. La falta de tiempo (llegar tarde a casa y sin terminar, trabajar en casa), el cansancio, pero sobre todo el impacto emocional (nerviosismo, insatisfacción, enfado) tienen una alta relación positiva con disciplina apropiada y sobre todo con una pobre de supervisión. También se encontró una correlación significativa y moderada entre esta variable y la disciplina severa.

Con respecto a la relación entre las variables de conciliación familia - trabajo y la sintomatología externalizante, tal y como puede observarse, se obtuvieron correlaciones significativas y moderadas fundamentalmente entre las conductas problemáticas que implican ruptura de normas y la tensión emocional que acompaña al conflicto trabajo-familia. Como se observa en la tabla 3, se asoció la presencia de conductas antisociales de manera más intensa con llegar con enfado o nerviosismo a casa y, aunque con intensidad menor, también se observan correlaciones significativas y moderadas con llegar tarde y cansado/a, llegar tarde y sin terminar o con quejarse del trabajo. La conciliación tuvo una correlación menor con las conductas agresivas de los y las adolescentes.

Una vez analizada la relación bivariada entre todas las variables se analizó el papel mediador de las variables familiares en la relación entre la conciliación y la sintomatología externalizante. A nivel de funcionamiento familiar se tuvieron en cuenta dos variables, 
Tabla 3

Correlación entre conciliación familia- trabajo y sintomatología externalizante (agresividad y ruptura de normas)

\begin{tabular}{|l|c|c|}
\hline & Agresividad & Ruptura Normas \\
\hline Cambiar planes & & $.24^{\star}$ \\
Llegar tarde a casa y sin terminar & $.32^{\star *}$ \\
Llegar nerviosos/as & $.27^{*}$ & $.46^{* *}$ \\
Trabajar en casa & $.25^{*}$ & $.49^{* *}$ \\
Llegar enfadados/as & & $.32^{\star *}$ \\
Quejarse del trabajo & $.35^{\star *}$ \\
\hline Llegar tarde y cansados/as & & \\
\hline $\begin{array}{l}\text { Nota. Se reportan únicamente los coeficientes significativos, en orden a facilitar la lectura de la tabla. } \\
* p<.01 \text { "* } p<.001\end{array}$ & \\
\hline
\end{tabular}

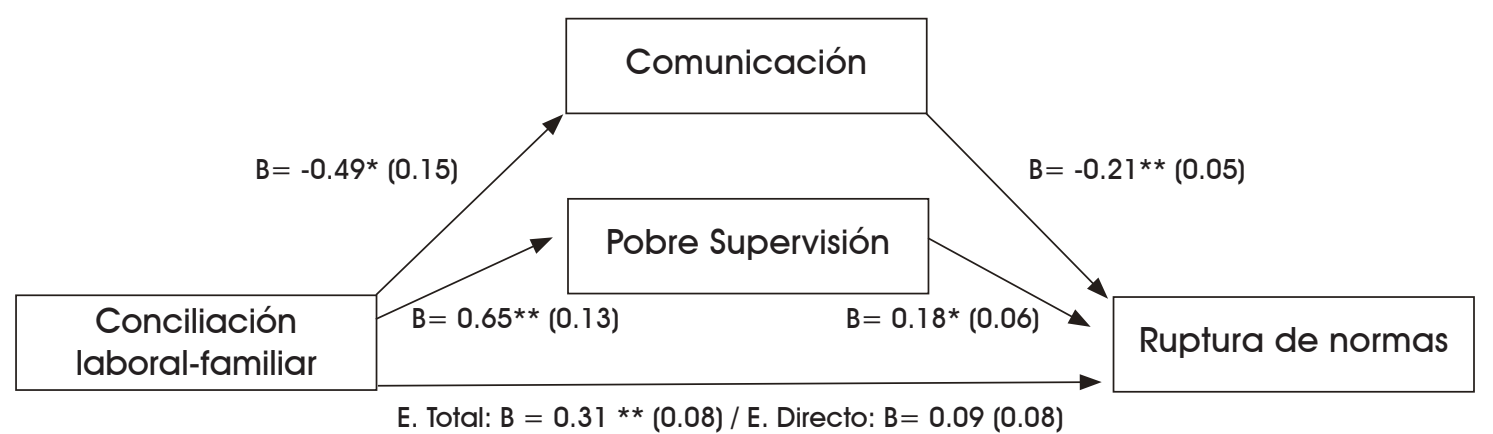

E Indirecto Global: 0.22 (I.C. 95\%: $0.10 / 0.35)$

E Indirecto Comunicación: 0.10 (I.C. 95\%: 0.03 / 0.21)

E. Indirecto No Supervisión: 0.12 (I.C. 95\%: 0.04 / 0.23)

Fig 1. Análisis del efecto mediador de la comunicación y de la no supervisión en la relación entre el conflicto familiar - laboral

(medido en sentido negativo, denominándose "conciliación") y la ruptura de normas.

${ }^{*} \mathrm{p}<.01,{ }^{* *} \mathrm{p}<.001$.

una vinculada al funcionamiento familiar global y la otra al estilo parental: comunicación y supervisión, las cuales fueron destacadas por su mayor asociación con la conciliación en los resultados previos. A nivel de sintomatología externalizante, se analizó exclusivamente, la ruptura de normas, por haber sido la variable más vinculada con las dificultades de conciliación. Los resultados de la mediación se pueden observar en la Figura 1. Tal como se puede comprobar, se verificó el efecto de mediación por parte de ambas variables mediadoras, así como por parte del modelo en global (ambos mediadores en conjunto). El tamaño de efecto de la mediación fue grande $\left(f^{2}=.44\right)$, y las variables independiente y mediadoras explicaron una cantidad significativa de varianza de la variable dependiente $\left(R^{2}=.43, p<.001\right)$. Al observar el efecto indirecto de cada una de las variables mediadoras, se observa que ambas tuvieron un efecto similar como mediadoras.

\section{DISCUSIÓN}

El objeto de este estudio ha sido comprobar el papel del conflicto familiar en la sintomatología externalizante de los y las adolescentes, analizando el papel mediador de las variables familiares.

En primer lugar, y tras comprobar la alta frecuencia de percepción de conflicto entre las demandas laborales y familiares, se estudiaron las asociaciones entre la tensión y sobrecarga laboral, por un lado, y el clima familiar (funcionamiento familiar y estilos educativos), por el otro. Se encontraron correlaciones 
significativas muy interesantes. En primer lugar, a nivel de funcionamiento familiar, la variable con la que se observa una mayor asociación es la comunicación. Esto parece relacionarse con la menor implicación, comunicación o compromiso en la vida familiar, observada en relación a la falta de tiempo en los estudios de llies et al. (2007) o Jacobs y Kelley (2006). También, en concordancia con esta idea parece que la insatisfacción en el trabajo podría generalizarse y llevar a una menor satisfacción familiar.

A nivel de pautas educativas, la variable más destacada fue la de supervisión, la cual apareció especialmente relacionada con la sobrecarga emocional (por nerviosismo, enfado, cansancio, etc.) o tensión. A tenor de los resultados, esta sobrecarga emocional parece relacionarse: (a) de forma negativa con la disciplina apropiada y de forma positiva con una disciplina severa: explicable desde la hipótesis de la hostilidad y (b) con la inconsistencia en la disciplina: explicable desde la disminución de la competencia parental.

Nuevos análisis de correlación permitieron observar la alta asociación existente entre dificultades de conciliación y sintomatología externalizante de los hijos/as. La correlación es especialmente importante con respecto a la variable "Ruptura de normas".

Por tanto, si bien es fundamental a nivel familiar generar, siempre, un ambiente estable y sensible, en este periodo evolutivo, de gran inestabilidad a muchos niveles (físico, cognoscitivo y emocional), es especialmente importante. El crecer y desarrollarse en medio de regularidad, afecto y comunicación, son fundamentales para el/la adolescente, con el fin de ir ganando el nivel de responsabilidad y autonomía que necesita. Por el contrario, la tensión generada por la sobrecarga laboral, hace a los progenitores menos sensibles a sus necesidades, menos proclives a apoyar e implicarse en su educación y más inconsistentes en la disciplina, combinando con frecuencia la falta de atención y la severidad.

La falta de consistencia en las normas, la falta de supervisión, hace difícil el aprendizaje de las reglas de conducta y la falta de tiempo adecuado de comunicación impiden generar marcos desde los que organizar reglas que rijan su conducta.

Estas condiciones no favorecen el desarrollo de seguridad emocional, autoestima positiva, independencia conductual o competencia social que destaca Belsky (1984) como fundamentales en su desarrollo.

Con respecto a la visión sistémica, el presente estudio parece mostrar un cierto respaldo para (a) la hipótesis de la transferencia de la hostilidad del trabajo a la familia, pues se encuentra relación entre quejarse del trabajo e insatisfacción familiar; (b) la hipótesis del deterioro de las prácticas de crianza; y (c) para la hipótesis de la disminución del afecto.

En relación a los análisis de mediación, debemos destacar la existencia de mediación múltiple de la comunicación y supervisión, lo cual nos indica que las dificultades de comportamiento (la activación emocional negativa, asociada a la agresividad, y el mal comportamiento o ruptura de normas) están vinculadas en su totalidad a las prácticas de disciplina y a los estilos de crianza. De forma que, incluso, en situaciones de intenso trabajo, si las figuras parentales son capaces de mantener un estilo de crianza afectuoso y consistente, no encontraríamos relación entre las dificultades de conciliación y la sintomatología externalizante de los/las adolescentes.

En conclusión, la familia, como unidad es dinámica. Ha variado de manera importante su forma, en las últimas décadas, pero desde un principio de equifinalidad, al margen de la diversidad de estructuras familiares, la idoneidad de las familias dependerá o no del cumplimiento de funciones. No obstante, los resultados de este estudio parecen ilustrar que igualitarismo y conciliación no han sido abordados con seriedad.

La incorporación femenina al mundo laboral ha supuesto una menor presencia de la mujer en el hogar en su papel tradicional (Ababkov et al. 2005; Ayree et al. 2005) sin que (de forma global) se haya producido una reorganización familiar que compatibilice la vida familiar y laboral. Y, sin dicha reorganización, sólo asistiremos a las consecuencias negativas, siendo los principales perjudicados los hijos e hijas.

Reconocido por los propios adolescentes (Finley et al. 2008), los padres y madres son los más relevantes contribuidores a su desarrollo. No obstante, esto implica que asistimos a la necesidad social de cambiar la forma de conciliar trabajo y la familia.

Es importante destacar que las afirmaciones en base a los resultados de este estudio deben abordarse con cautela, pues se trata de un 
acercamiento al estudio de la conciliación, el cual deberá replicarse en el futuro con una muestra mayor que incorpore tanto a los progenitores como a sus hijos e hijas.

\section{- Conflicto de intereses}

Los autores declaran no tener ningún conflicto de intereses.

\section{REFERENCIAS}

Ababkov, V. A., Perrez, M., Kaidanovskaia, E. V. y Shiobi, D. (2005). Family life and professional activity. Russian education and society, 47 (12), 5- 20.

Achenbach, T. M. (1991) Integrative guide to the 1991 CBCL/4-18, YSR, and TRF profiles. Burlington, VT: University of Vermont, Department of Psychology.

Alpert, J. S. (2010). Balancing work, family and friends, and lifestyle. American Journal of Medicine, 123(9), 775-776.

Aryee, S., Srinivas, E. S. y Tan, H. H. (2005). Rhythms of life: antecedents and outcomes of work-family balance in employed parents. Journal of applied psychology, 90 (1), 132 146.

Barnes, H. L. y Olson, D. H. (1985). Parentadolescent communication and the Circumplex model. Special issue: Family development. Child Development, 56, 438-447.

Barnes, H. L., \& Olson, D. H. (1982). Parentadolescent communication scale. En D. H. Olson, H. McCubbin, H. L. Barnes, A. Larsen, M. Muxen y M. Wilson (Eds.), Family inventories: Inventories used in a national survey of families across the family life cycle (pp. 51 -63). St. Paul, MN: University of Minnesota.

Belsky, J. (1984). The determinants of parenting: A process model. Wiley-Blackwell. doi:10.1111/1467-8624.ep7405453

Carrasco, C. (2001). La sostenibilidad de la vida humana: ¿̇un asunto de mujeres?. Mientras tanto, 86, 1-27.

Ceballos, E. (2006). Dimesiones de análisis del diagnóstico en educación: El diagnóstico del contexto familiar. RELIEVE, 12 (1), 33-47.

Cohen, J. (1988). Statistical power analysis for the behavioral sciences (2nd ed.). Hillsdale, NJ: Erlbaum.
Crum, K. I., \& Moreland, A. D. (2017). Parental stress and children's social and behavioral outcomes: The role of abuse potential over time. Journal of Child and Family Studies, 26(11), 3067-3078.

Dadds, M. R., Maujean, A., y Fraser, J. A. (2003). Parenting and conduct problems in children: Australian data and psychometric properties of the Alabama Parenting Questionnaire. Australian Psychologist, 38, 238-241.

Davies, P. T. y Cummings, E. M. (1994). Marital conflict and child adjustment: An emotional security hypothesis. Psychological bulletin, 116 (3), 387-41 1.

Finley, G., Mira, S. y Schwartz, S. (2008). Perceived paternal and maternal involvement: Factor structures, mean differences, and parental roles. Fathering, 6 (1), 62- 82.

Ford, M. T., Heinen, B. A. y Langkamer, K. L. (2007). Work and family satisfaction and conflict: A meta-analysis of cross-domain relations. Journal of applied psychology, 92, (1), 57- 80 .

Frick, P. J. (1991). The Alabama Parenting Questionnaire. Unpublished Unpublished rating scale. University or Alabama.

Golden, T. D., Veiga, J. F. y Simsek, Z. (2006). Telecommuting's differential impact on workfamily conflict: Is there no place like home? Journal of applied psychology, 91 (6), 1340 1350.

Gonzalo, D. C. (2016). Influence of Cushioning Variables in the Workplace and in the Family on the Probability of Suffering Stress. Safety and Health at Work, 7 (3), 175-184.

Hayes, A. F. (2013). Introduction to mediation, moderation, and conditional process analysis: A regression-based approach. New York: Guilford Press.

Honeycutt, J. M., Sheldon, P., Pence, M. E., \& Hatcher, L. C. (2015). Predicting Aggression, Conciliation, and Concurrent Rumination in Escalating Conflict. Journal of Interpersonal Violence, 30(1), 133-151.

Ilies, R., Schwind, K. M., Wagner, D. T., Johnson, M. D., DeRue, D. S. y llgen, D. R. (2007). When can employees have a family life? The effects of daily workload and affect on work-family 
conflict and social behaviors at home. Journal of applied psychology, 92 (5), 1368- 1379.

Jacobs, J. M. y Kelley, M. L. (2006). Predictors of paternal involvement in childcare in dualearner families with Young children. Fathering, 4, (1), 23- 47.

Livingston, B. A. y Judge, T. A. (2008). Emotional responses to work-family conflict: An examination of gender role orientation among working men and women. Journal of applied psychology, 93, (1), 207- 216.

Lohman, B. J., Newman, B. M., y Newman, P. R. (2007) Peer group membership and a sense of belonging: their relationship to adolescent behavior problems. Adolescence, 42, 241 263.

López, M. T., Utrilla, A. y Valiño, A. (2006). Políticas públicas y familia. Análisis de la situación en España. Fundación Acción Familiar, Fundación Gondara Barandiarán. Madrid: Ed. Cinca.

Low, S. M., y Stocker, C. (2005). Family Functioning and Children's Adjustment: Associations Among Parents' Depressed Mood, Marital Hostility, Parent-Child Hostility, and Children's Adjustment. Journal Of Family Psychology, 19 (3), 394-403. doi:10.1037/08933200.19.3.394

Martínez-Pampliega, A., Iraurgi, I., Galíndez, E., y Sanz, M. (2006). Family Adaptability and Cohesion Evaluation Scale (FACES): Desarrollo de una versión de 20 ítems en español. International Journal of Clinical and Health Psychology, 6, (2), 317-338.

Matias, M., \& Fontaine, A. M. (2014). Managing Multiple Roles: Development of the WorkFamily Conciliation Strategies Scale. Spanish Journal of Psychology, 17 (2), e56.

Olson, D. H. y Wilson, M. (1982). Family Satisfaction. En D. H. Olson, H. I. McCubbin, H. Barnes, A. Larsen, M. Muxen y M. Wilson (Eds.), Family inventories: Inventories used in a national survey of families across the family life cycle (pp. 43-49). St. Paul, MN: University of Minnesota.
Olson, D. H., Portner, J., Bell, R.Q. (1982). FACES-II: Family Adaptability and Cohesion Evaluation scales. St. Paul, MN: Family Social Science, University of Minnesota.

Olson, D. H., Stewart, K. L., y Wilson, L. R. (1990). Health and stress profile (HSP), revised. Minneapolis: Profile of Health Systems.

Sanz, C. M. (2003). The family dynamics of drug addicts who are under treatment. Tesis doctoral. Universidad de Deusto (Bilbao). Disponible en la base de datos Dissertations \& Theses@ Universidad de Deusto.

Sanz, M., Iraurgi, I., y Martínez-Pampliega, A. (2002). Evaluación del funcionamiento familiar en toxicomanías: Adaptación española y características de adecuación métrica del FAP-FACES IV. En I. Iraurgi y F. GonzálezSaiz (Eds.), Instrumentos de evaluación en drogodependencias (pp. 403-434). Madrid: Aula Médica.

Scharlach, A. E. (2001). Role strain among working parents: implications for workplace and community. Community, work \& family, 4 (2), 215- 230.

Serrano- Manríquez, M. (2007). Maltrato infantil: variables sociodemográficas y prácticas de crianza. Ciencia psicológica, 1, (2), 55- 63.

Servera, M. (2007). Versión en Español del Alabama Parenting Questionnaire (APQ). Versión para padres. Documento no publicado. Spector, P.E., Allen, T. D., Poelmans, S. A. Y., Lapierre, L. M., Cooper, C. L., O'Driscoll, M., Sanchez, J. I., Abarca, N., Alexandova, M., Beham, B., Brough, P., Ferreiro, P., Fraile, G., Lu. C., Lu, L., Moreno-Velázquez, I. y Pagon, M. (2007). Cross-national differences in relationships of work demands, job satisfaction, and turnover intentions with work-family conflict. Personnel Psychology, 60, 805- 835.

Unger, D. G., Brown, M. B., Tressell, P. A. y McLeod, L. E. (2000). Interparental Conflict and Adolescent Depressed Mood: The Role of Family Functioning. Child Psychiatry \& Human Development, 31, 1, 23-41. 